\title{
A case report of intestinal obstruction caused by cryptogenic multifocal ulcerous stenosing enteritis
}

\author{
Cheng Chang ${ }^{1}$, Chen Jiang ${ }^{2}$, Yaoyao Miao ${ }^{3}$, Bin Fang ${ }^{4}$ and Lili Zhang ${ }^{5^{*}}$ (D
}

\begin{abstract}
Background: Cryptogenic multifocal ulcer stenosing enteritis (CMUSE) is a rare disease characterized by multiple superficial ulcers, stenosis, and obstruction of the small intestine of unknown origin, and the course can recur.

Case presentation: We encountered a 62-year-old male patient with intestinal obstruction. The patient was admitted to the hospital for surgical treatment due to intestinal obstruction, and was diagnosed with cryptogenic multifocal ulcer stenosis enteritis due to comprehensive surgery and postoperative pathological considerations.
\end{abstract}

Conclusion: In the future, we will continue to follow up the patient. The present study aims to remind clinicians of this disease, and reduce the incidence of misdiagnosis.

Keywords: Cryptogenic multifocal ulcer stenosing enteritis, Intestinal obstruction, Small intestinal ulcer, Small intestinal stenosis

\section{Background}

Cryptogenic multifocal ulcerous stenosing enteritis (CMUSE) is a rare idiopathic disease, At present, there are only over 60 cases of CMUSE reported in the world [1], this was first reported in 1964 [2]. This is featured by unexplained small bowel multiple superficial ulcers, stenosis, obstruction, and no biological signs of systematic inflammation, which are prone to recurrence. Hormone therapy is effective for some patients [3]. We encountered a patient with intestinal obstruction, which was very severe, and this patient was admitted to the hospital for immediate surgical treatment. Combined with the intraoperative findings and postoperative pathological diagnosis, the patient was diagnosed as CMUSE.

\footnotetext{
* Correspondence: zhang020361@sina.com

${ }^{5}$ Department of Radiology, The Affiliated Qingdao Hiser hospital of Qingdao University (Qingdao Hospital of Traditional Chinese Medicine ), Renmin Road 4, Qingdao 266033, Shandong Province, China

Full list of author information is available at the end of the article
}

\section{Case presentation}

A 62-year-old male patient was admitted to the hospital due to "abdominal pain and abdominal distension for one month, with aggravation for five days". The patient presented with abdominal pain in the previous one month without obvious inducement, mainly around the umbilicus, paroxysmal, accompanied by abdominal distension, with a small amount of exhaust and defecation. The patient continued to eat without paying attention to this. Five days ago, these symptoms aggravated, and the patient stopped venting and defecating. The patient visited our hospital for treatment and underwent abdominal CT, which revealed small intestinal obstruction (Fig. 1). Physical examination: abdominal distention, intestinal type, total abdominal tenderness, obvious periumbilical, and bowel sounds hyperactive. Laboratory examination: WBC count: $19.61 \times 10^{9} / \mathrm{L}$, hemoglobin: $91 \mathrm{~g} / \mathrm{L}$, serum albumin $22 \mathrm{~g} / \mathrm{L}$, and the remaining assay examination was normal. Previous history: good health, no history of abdominal surgery, and no history of 


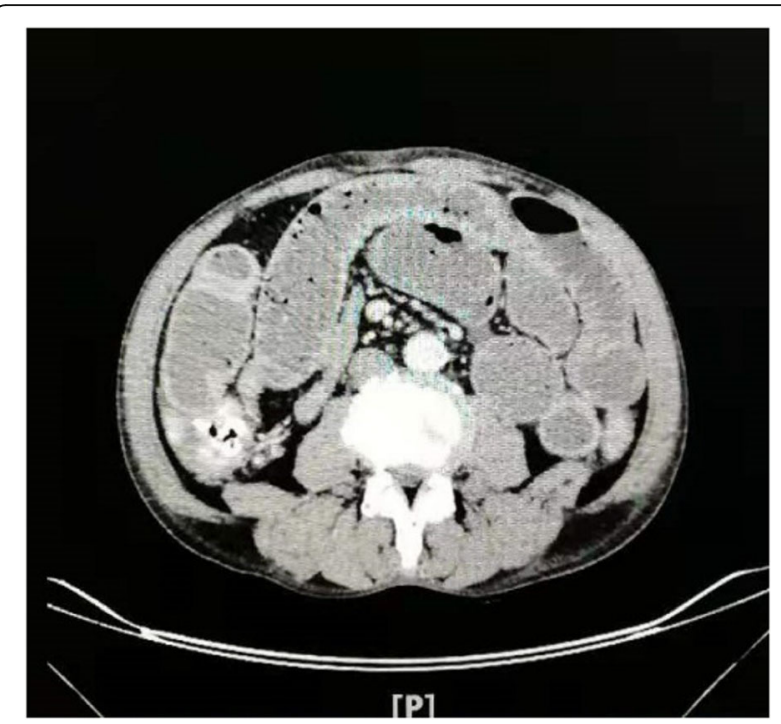

Fig. 1 Preoperative abdominal CT examination showed small bowel obstruction

medication. Since the obstruction was long and severe, emergency laparotomy was performed.

Observations during the operation: there was no adhesion in the abdominal cavity, there was no twist and compression of the intestine, no tumor was detected, and the obstruction was located in the small intestine at $70 \mathrm{~cm}$ away from the ileocecal area. The small intestine above the obstruction was highly dilated, and the diameter of the intestine was $8-10 \mathrm{~cm}$. The appearance of the following small intestine was normal. The intestinal canal was longitudinally dissected at the obstruction, a small amount of food fiber was expelled from the intestinal canal at the obstruction site, and a "stenosis ring" was formed in the small intestinal cavity. Merely pores with a diameter of $0.3-0.4 \mathrm{~cm}$ were left, and no obvious abnormality was observed in the intestinal mucosa (Fig. 2). The distal intestine was longitudinally opened to the ileocecal region, and multiple "stenosis rings" were found in the intestinal lumen at approximately $40 \mathrm{~cm}$ from the distal obstruction, presenting with the same shape (Fig. 3). The shortest spacing between the "stenosis ring" was $1.0 \mathrm{~cm}$, and the longest spacing was $6 \mathrm{~cm}$. The diseased bowel was removed, and the patient recovered well without discomfort, such as diarrhea. The patient was followed up for two months, and no discomfort was found.

Pathological findings included: intestinal stenosis was obtained, shallow intestinal segmental ulcer formation, mucosal erosion and inflammatory granulation tissue formation, the mucosal muscle layer disappeared, submucosal fiber hyperplasia, and longitudinal extension to the mucosal layer, causing mucosal uplift and intestinal wall stenosis A large amount of neutrophil infiltration, and vasodilatation and congestion were observed in the

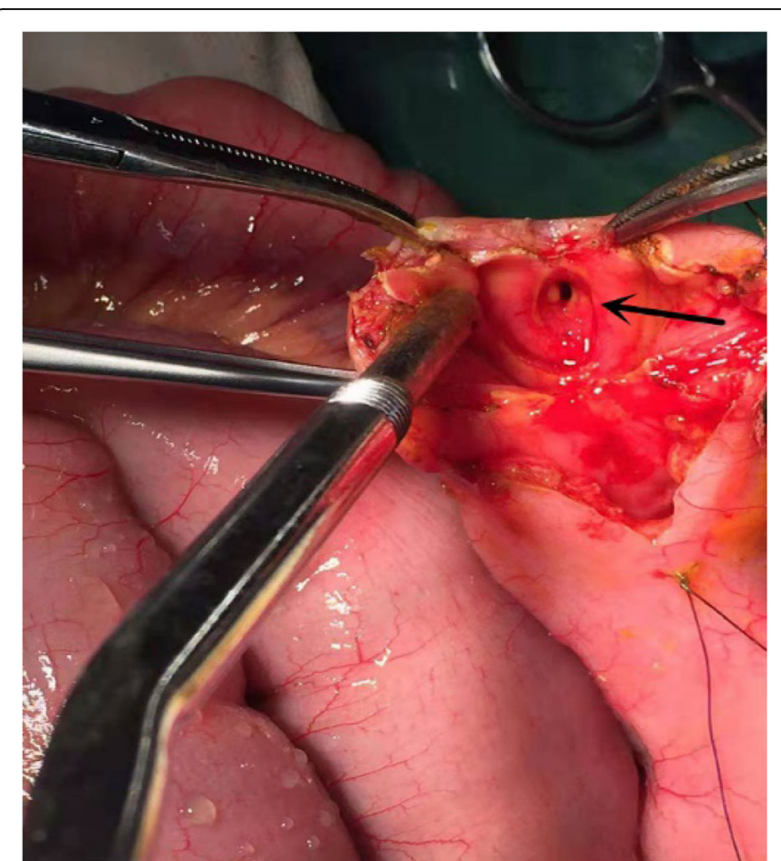

Fig. 2 Intraoperative photograph. The black arrow shows " stenosis ring "

serosa, but no obvious structural abnormalities were found in the muscle layer and serosa layer (Fig. 4).

\section{Discussion and conclusion}

The etiology and pathogenesis of CMUSE remains unknown. Some scholars speculate that autoimmune

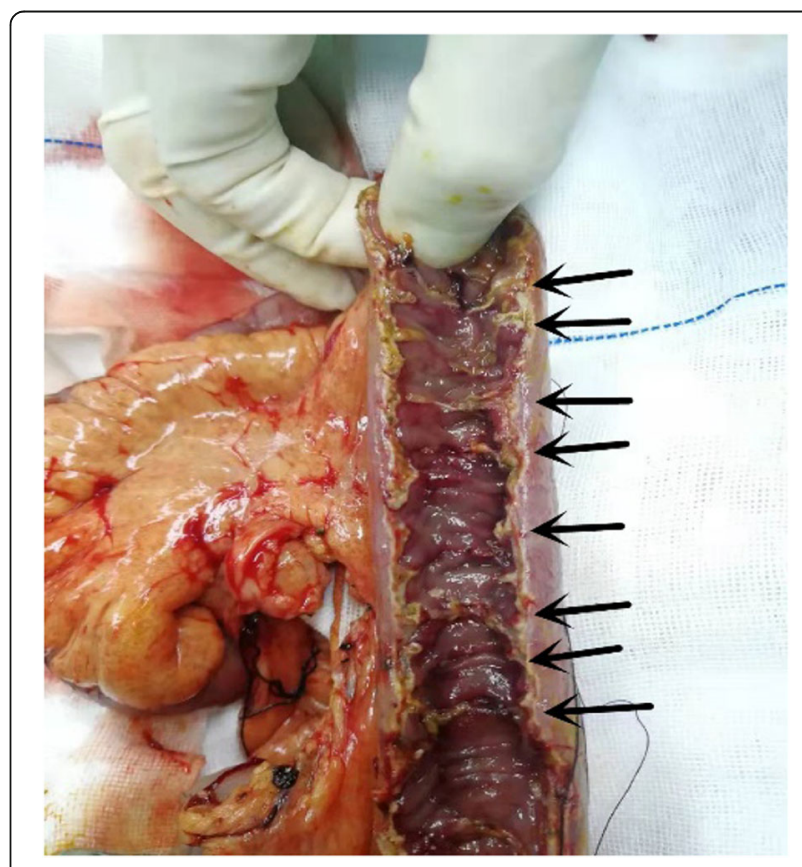

Fig. 3 Intraoperative photograph. The black arrow shows " stenosis ring "(Has been opened) 


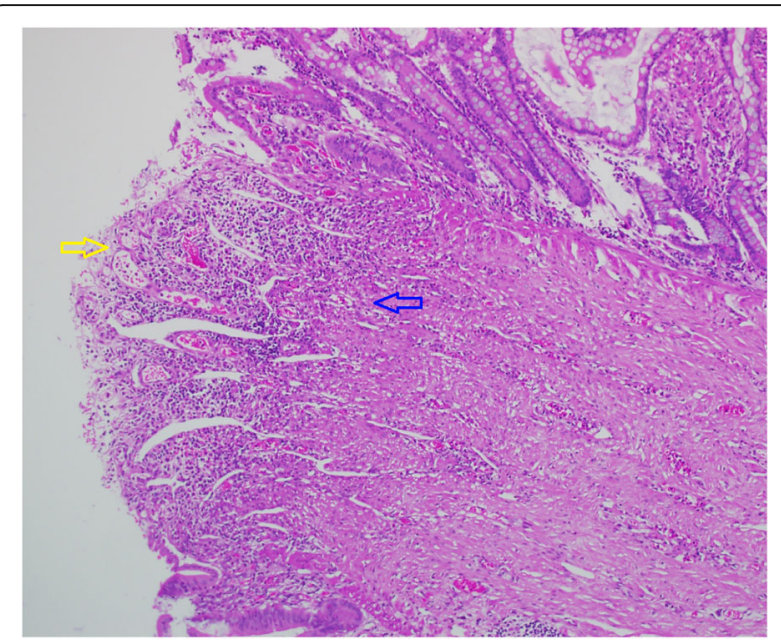

Fig. 4 Postoperative pathological photos. HE stain, 100 times magnification. Yellow arrow: shallow intestinal mucosa ulcer. Blue arrow: muscle fibers growing longitudinally below the ulcer.

abnormalities, excessive formation of fibrous tissue, vasculitis, and mutations in the gene PLA2G4A encoding cytosolic phospholipase A2-a are correlated to its pathogenesis [3, 4].

Yu Zhang et al. [5] summarized the diagnostic criteria for CMUSE: (1) Medical history: long course; persistent and occult blood loss from the gastrointestinal tract with severe anemia. (2) Clinical features: chronic and relapsing subileus episodes; chronic iron-deficiency anemia with fatigue, edema, or growth retardation, rarely with diarrhea; with normal inflammatory makers or other biological signs of systemic inflammation; normal colon and stomach; no extrointestinal manifestations. (3) Imaging manifestation: In enteroclysis: multiple persisting stenoses of the small intestine caused by brous strictures. (4) Endoscopic findings: multiple, pleiomorphous and superficial ulcers, and sharply demarcated from the surrounding normal mucosa; caution was taken during the capsule endoscopy examination, because this may become stranded in the strictures. (5) Histological findings: necrotic inflammatory ulcers not reaching the proper muscular layer; nonspecific inflammation, and erosion with submucosal fibrosis.

Differential diagnosis could include: (1) Crohn's disease (CD): $\mathrm{CD}$ is one of the most important differential diagnoses for CMUSE. The difference is that Crohn's disease is transmural inflammation, and the typical lesions are longitudinal and fissured ulcers, which are prone to fistula formation and intestinal perforation. Giant cell granulomas are observed in its pathology [6]. (2) NSAID-associated bowel disease: This disease was very similar to CMUSE under endoscopy, with superficial ulcers and intestinal stenosis [7], but NSAID-associated bowel disease has a long history of medication, and can be recovered after withdrawal [8]. (3) Intestinal tuberculosis can occur with intestinal ulcers or stenosis. Its characteristics include the following: circular ulcers, which can be accompanied by extraintestinal tuberculosis; the histopathological findings of caseous necrotizing granulomatous or positive acid staining could be confirmed. (4) Vasculitis involves multiple ulcers in the intestine, such as Behcet's disease, granulomatous vasculitis, and systemic scleroderma [9]. However, these ulcers are deep, and may have perforations. Furthermore, multifocal stenosis is rare, and blood vessel inflammation is a systemic disease that often involves multiple systems.

Main treatment methods includes: (1) Hormones: Glucocorticoids are presently the first line of treatment, but these are likely to cause hormone dependence, relapse, and steroid resistance [7]. (2) Endoscopic balloon dilatation: This can alleviate the symptoms, and prevent small bowel resection [3, 4]. (3) Surgery: This can easily relapse after surgery, and repeated surgery can easily cause complications, such as short bowel syndrome [3]. (4) Nutritional support therapy: Enteral or parenteral nutrition and iron supplementation can improve the symptoms in the short term, and even obtain mucosal healing. However, since the patient resumed eating, the mucosal ulcer, anemia and hypoproteinemia rapidly relapsed [10].

CMUSE is a rare disease of unknown etiology. Multiple superficial ulcers and intestinal stenosis in the small intestine are its typical manifestations. Its diagnosis depends on the comprehensive judgment of clinical manifestations, imaging examinations, endoscopy and pathological examinations. The investigators will continue to follow the patient and monitor for its recurrence. The present study aims to remind clinicians of this disease. When encountering similar lesions, the possibility of this disease must be considered. This also provides a differential diagnosis for small bowel ulcers and stenotic diseases, reducing the occurrence of misdiagnosis.

\section{Abbreviations \\ CMUSE: Cryptogenic multifocal ulcer stenosing enteritis; CT: Computer tomography; WBC: White blood cell count; $\mathrm{Cm}$ : Centimeters; CD: Crohn's disease; NSAIDs: Non-Steroidal Anti-inflammatory Drugs}

\section{Acknowledgements \\ We would like to acknowledge Dr. Jie Lu for the images taken during the operation.}

\section{Authors' contributions}

$\mathrm{CC}$ and $\mathrm{BF}$ were involved in the treatment of the patient described in the case report, and initiated the case report. CC,BF,YYM and LLZ prepared the first draft of the manuscript. CJ provided the pathological information. All authors participated in further drafting and revision of the manuscript. All authors have read and approved the final manuscript.

\section{Funding}

There are no funding sources for this case report. 


\section{Availability of data and materials}

This case report contains clinical data obtained from the electronic medical record in Qingdao Municipal Hospital (Group). Additional information is available upon request, but only in accordance with the privacy restrictions of the hospital.

\section{Ethics approval and consent to participate}

Not applicable.

\section{Consent for publication}

An informed consent was obtained from the patient. Verbal and written consents were obtained for the publication of the case report and the accompanying images. A copy of the written consent is available upon request.

\section{Competing interests}

The authors declare that they have no competing interests.

\section{Author details}

'General Surgery, Qingdao Municipal Hospital (Group), Qingdao 266011, Shandong Province, China. ${ }^{2}$ Pathology Department, Qingdao Municipal Hospital (Group), Qingdao 266011, Shandong Province, China. ${ }^{3}$ Infectious Diseases Department, The Affiliated Qingdao Hiser hospital of Qingdao University (Qingdao Hospital of Traditional Chinese Medicine ), Qingdao 266033, Shandong Province, China. ${ }^{4}$ Department Of Anus \& Intestine Surgery, The Affiliated Qingdao Hiser hospital of Qingdao University, (Qingdao Hospital of Traditional Chinese Medicine ), Qingdao 266033, Shandong Province, China. ${ }^{5}$ Department of Radiology, The Affiliated Qingdao Hiser hospital of Qingdao University (Qingdao Hospital of Traditional Chinese Medicine ), Renmin Road 4, Qingdao 266033, Shandong Province, China.

Received: 19 March 2020 Accepted: 11 September 2020

Published online: 15 September 2020

\section{References}

1. Kohoutova D, Bartova J, Tacheci I, et al. Cryptogenic multifocal ulcerous stenosing enteritis: a review of the literature. Gastroenterol Res Pract. 2013; 2013:918031.

2. Lindholmer B, Nyman E, Raef L. Nonspecific Stenosing Ulceration of the Small Bowel: A Preliminary Report. Acta Chir Scand. 1964;128:310-1.

3. Perlemuter G, Guillevin L, Legman P, Weiss L, Couturier D, Chaussade S. Cryptogenetic multifocal ulcerous stenosing enteritis: an atypical type of vasculitis or a disease mimicking vasculitis. Gut. 2001;48:333-8.

4. Kohoutova D, Bures J, Tycova V, et al. Severe cryptogenic multifocal ulcerous stenosing enteritis. A report of three cases and review of the literature. Acta Medica (Hradec Kralove). 2010;53:25-9.

5. Zhang $Y$, Huang $L$, Liu $R$, et al. Case report of a pair of siblings with cryptogenic multifocal ulcerating stenosing enteritis: A rare disease easily to be misdiagnosed as Crohn disease. Medicine (Baltimore). 2017;96:e7527.

6. Perlemuter $\mathrm{G}$, Chaussade $\mathrm{S}$, Soubrane $\mathrm{O}$, et al. Multifocal stenosing ulcerations of the small intestine revealing vasculitis associated with C2 deficiency. Gastroenterology. 1996;110:1628-32.

7. Matsumoto T, lida M, Matsui T, Yao T. Chronic nonspecific multiple ulcers of the small intestine: a proposal of the entity from Japanese gastroenterologists to Western enteroscopists. Gastrointest Endosc. 2007;66: S99-107.

8. Matsumoto T, lida M, Matsui T, et al. Non-specific multiple ulcers of the small intestine unrelated to non-steroidal anti-inflammatory drugs. J Clin Pathol. 2004:57:1145-50.

9. Ebert EC. Gastric and enteric involvement in progressive systemic sclerosis. J Clin Gastroenterol. 2008:42:5-12.

10. Esaki M, Umeno J, Kitazono T, Matsumoto T. Clinicopathologic features of chronic nonspecific multiple ulcers of the small intestine. Clin J Gastroenterol. 2015;8:57-62.

\section{Publisher's Note}

Springer Nature remains neutral with regard to jurisdictional claims in published maps and institutional affiliations.

\section{Ready to submit your research? Choose BMC and benefit from:}

- fast, convenient online submission

- thorough peer review by experienced researchers in your field

- rapid publication on acceptance

- support for research data, including large and complex data types

- gold Open Access which fosters wider collaboration and increased citations

- maximum visibility for your research: over $100 \mathrm{M}$ website views per year

At BMC, research is always in progress.

Learn more biomedcentral.com/submissions 